\title{
Diferenças de sexo na relação entre indicadores de força e resistência muscular de membros inferiores e a presença de incontinência urinária em nonagenários e centenários
}

\section{Gender differences in the relationship between lower limb muscle strength and resistance indicators and presence of urinary incontinence in nonagenarians and centenarians}

\author{
Luisa Braga Jorge', Josemara de Paula Rocha', Patricia Morsch², Bruna Borba Neves', \\ Gabriela Guimarães Oliveira' ', Ângelo José Gonçalves Bós' \\ Escola de Medicina da Pontifícia Universidade Católica do Rio Grande do Sul. Porto Alegre, RS, Brasil. \\ 2 Faculdade de Educação e Meio Ambiente (FAEMA). Ariquemes, RO, Brasil.
}

\section{ARTICLE INFO}

\section{Article history}

Received: 12/06/2018

Accepted: $11 / 10 / 2018$

\section{Correspondent Author}

Luisa Braga Jorge

Rua Domingos Crescêncio, 280/904

Bairro Santana

90650-090 Porto Alegre, RS, Brasil

(D) orcid.org/0000-0003-2695-8340

<lbragajorge@hotmail.com>

(C) 2018 All rights reserved

Editors

Alfredo Cataldo Neto

Paula Engroff

\begin{abstract}
RESUMO
OBJETIVOS: Verificar a possível relação entre incontinência urinária e indicadores de força e resistência de membros inferiores em nonagenários e centenários e a influência do sexo sobre a mesma. METODOS: Estudo transversal e analítico com nonagenários e centenários do projeto Atenção Multiprofissional ao Longevo. A incontinência, facilidade em executar atividades relacionadas aos membros inferiores, teste Timed Up and Go, sexo, idade, estado conjugal e sintomas depressivos foram as variáveis do estudo. Análise descritiva e analítica foi realizada pelos teste de qui-quadrado, ANOVA e regressão logística univariada e ajustada pelo sexo aceitando $p<0,05$. RESULTADOS: Foram avaliados 238 idosos, sendo 174 mulheres. A facilidade para caminhar 400 metros e levantar-se de uma cadeira e o tempo no Timed Up and Go foram os indicadores de força muscular e resistência significativos na correlação entre incontinência e força e resistência muscular ajustado pelo sexo (respectivamente, $R C=0,832$, $p=0,011 ; R C=0,846, p=0,012$ e $R C=0,352, p=0,002)$. Sexo foi a variável com relação mais significativa $(R C=0,349, p=0,001)$ e sua presença diminuía a significância estatística das demais variáveis. Sintomas depressivos aumentavam as chances de incontinência para homens. CONCLUSÕES: A incontinência pode estar relacionada com força e resistência muscular de membros inferiores, principalmente em mulheres. Exercícios de força e resistência envolvendo membros inferiores podem ser agregados aos programas tradicionais e reconhecidos de prevenção de incontinência.
\end{abstract}

DESCRITORES: Longevidade; incontinência urinária; força muscular; idoso de 80 anos ou mais.

\begin{abstract}
AIMS: To verify the possible relation between urinary incontinence and lower limbs strength and resistance indicators and the sex influence in this relation. METHODS: Cross-sectional and analytical study with nonagenarians and centenarians from "Multiprofessional Attention to the oldest-old" project. Presence of incontinence, the ease to walking and climbing stairs, Timed up and Go test, sex, age, marital status and depression symptoms were the study variables. Descriptive and analytical analysis (chi-square tests, analysis of variance and univariate and sex-adjusted logistic regression) were performed accepting $p<0.05$. RESULTS: We evaluated 238 oldest-old, 174 women. The ease of walking 400 meters and stand up from a chair and the time during the Timed Up and Go were significant indicators of muscle strength and resistance in the correlation between incontinence and muscle strength and resistance adjusted by sex (respectively, $R C=0.832, p=0.011, R C=0.846, p=0.012$ and $R C=0.352, p=0.002$ ). Sex was the most significantly related to incontinence $(R C=0.349, p=0.001)$ and its presence in the complete model decreased the significance of other variables. Depressive symptoms increase the chance of presenting incontinence in men. CONCLUSIONS: Incontinence can be associated with strength and resistance in lower limbs, mainly in women. Strength and resistance exercises focus on lower limbs can be added to traditional and recognized prevention programs to incontinence.

KEYWORDS: Longevity; urinary incontinence; muscle strength; aged - 80 and over.
\end{abstract}




\section{INTRODUÇÃO}

A incontinência urinária (IU) segundo a Sociedade Internacional de Continência é definida como a queixa de qualquer perda involuntária de urina. ${ }^{1}$ Estudos internacionais demonstram que sua prevalência pode chegar a 30\% em idosos residentes no domicílio e até 40 $-70 \%$ em idosos hospitalizados ou institucionalizados, sendo mais prevalente no sexo feminino aumentando significativamente com o avançar da idade, embora possa acontecer em qualquer fase da vida. ${ }^{2}$

Devido a sua alta prevalência, a IU é considerada um problema de saúde pública, que além de causar uma grande despesa econômica e, aumentar o risco de outras doenças pode reduzir a qualidade de vida dos portadores, levando ao aparecimento de sintomas depressivos como isolamento e baixa autoestima. Além disso, a IU aumenta expressivamente o risco de institucionalização, fraturas e fragilidade. ${ }^{3}$ A fragilidade é uma condição clínica muito prevalente especialmente nas pessoas com 80 anos ou mais (longevos) e é caracterizada principalmente pela diminuição da massa e da força muscular. ${ }^{4}$ A perda da força da musculatura do assoalho pélvico é uma das principais causas de IU em longevos. ${ }^{5}$

Pesquisas relatam à correlação da IU e declínio funcional, assim como com a força muscular especialmente de membros inferiores (MMII), indicando que quanto maior a perda de força muscular, maior a severidade dos sintomas de IU. ${ }^{6,7}$ A força de MMII, por sua vez, está associada ao desempenho das atividades de vida diária e velocidade da marcha, estando relacionada também à incapacidade nas idades mais avançadas. ${ }^{8}$ Existem diversas maneiras de mensurar a força de MMII, incluindo o autorrelato do desempenho das atividades de vida diária e testes funcionais, como o Timed Up and Go (TUG)..$^{9}$ A maioria dos estudos sobre IU e o declínio funcional é restrita à população feminina. Por isso poucos estudos buscam avaliar a comparação do desempenho funcional e a IU entre homens e mulheres. Os homens apresentam distintas características funcionais e de envelhecimento podendo apresentar também distintas relações aos sintomas da IU. ${ }^{10}$ Com relação à força de MMII tanto homens quanto mulheres demonstram o mesmo padrão de diminuição muscular durante o envelhecimento. ${ }^{11}$ A mobilidade funcional e o TUG diminuem com o envelhecimento tanto em homens, quanto em mulheres, estudos mostram que essa diminuição não diferente entre os sexos. ${ }^{12}$

Dada à relevância da IU e da diminuição da força e resistência muscular em longevos, e principalmente nonagenários e centenários, urge a necessidade de verificar a possível relação entre os indicadores de força e resistência muscular de membros inferiores e a presença de IU em nonagenários e centenários. Além de investigar diferenças de sexo nessa problemática que possam influenciar na abordagem da IU.

\section{MÉTODOS}

Trata-se de um estudo transversal e analítico. O estudo integra parte do Projeto de Atenção Multiprofissional ao Longevo (AMPAL) da Pontifícia Universidade Católica do Rio Grande do Sul. O AMPAL é um estudo longitudinal aprovado pelo Comitê de Ética em Pesquisa da PUCRS pelo parecer número 1.639.961.

A técnica de amostragem foi de conglomerados representativos dos bairros de Porto Alegre, Rio Grande do Sul. Os participantes deveriam ter 90 anos ou mais, ser domiciliados em Porto Alegre e não residir em instituições de longa permanência, sendo incluídos idosos com qualquer nível funcional.

O processo de seleção dos participantes envolveu um estudo geográfico da cidade. A cidade de Porto Alegre está dividida em regiões do orçamento participativo desde 1997 e pode ser analisada através do sistema online disponibilizado pelo ObservaPOA. ${ }^{13}$ Combinando essas informações com as do IBGE Cidades do censo de 2010, foi possível separar essas grandes regiões em setores com 6 nonagenários. Esses setores foram sorteados usando o software Excel da Microsoft 2013 de modo a representar cada uma das regiões do orçamento participativo. A partir do sorteio, grupos de alunos identificadores realizaram busca ativa dos possíveis longevos moradores desses locais. Dessa identificação criou-se uma lista de longevos distribuídos por setores, que receberam ligações telefônicas para agendar as avaliações de acordo com o aceite. Nessas visitas eram apresentados os termos de consentimento livre e esclarecido para confirmar ou refutar a participação do longevo na pesquisa. Essa etapa da coleta dos dados iniciou em janeiro de 2016 e terminou em dezembro de 2016. Contudo o projeto continua em andamento.

Os participantes foram divididos em dois grupos: continentes (sem incontinência) e incontinentes (com incontinência, sendo a variável dependente do estudo e representada pelo autorrelato de perda urinária nos últimos seis meses).

Os indicadores de força e resistência muscular de membros inferiores foram a facilidade ou dificuldade em levantar-se de uma cadeira uma vez (força), caminhar 400 metros, independente do uso de dispositivos auxilidares (resistência), subir 10 degraus (resistência) 
e o TUG (resistência). O grau de facilidade foi avaliado em uma escala de quatro níveis autorrelatados pelos longevos: não consegue, difícil, \pm fácil e fácil. O TUG consistiu em cronometrar o tempo gasto na tarefa de levantar-se de uma cadeira (a partir da posição sentada), caminhar 3 metros, girar e voltar andando no mesmo percurso, sentando-se novamente com as costas apoiadas. ${ }^{14}$

Outras variáveis utilizadas e consideradas de controle foram: sexo, idade (em anos), estado conjugal (Casado, Solteiro/divorciado e Viúvo) e presença de sintomas depressivos. A presença de sintomas depressivos foi avaliada pela Escala de Depressão Geriátrica de 5 questões, sendo utilizada na análise estatística de variância seu escore e na regressão logística, a presença ou ausência de sintomas de acordo com o ponto de corte. Essa escala consiste em cinco perguntas sobre a presença de sintomas depressivos, sendo 2 pontos a pontuação de corte para estabelecer a presença desses sintomas, mostrando equivalência na eficácia quando comparado ao GD15..$^{15-16}$

Para a análise estatística foram realizados testes de Qui-quadrado para comparar as variáveis categóricas e testes de análise de variância para verificar a presença de relação entre a variável dependente e as médias das variáveis numéricas independentes. Foram realizados testes de regressão logística múltipla e univariada para identificar as variáveis relacionadas com a chance de ter incontinência, de modo independente e testes de regressão logística ajustada pelo sexo, para cada uma das demais variáveis, observando o efeito sobre cada uma delas na regressão. O programa estatístico utilizado foi o Epi info ${ }^{\text {TM }} 7.2$ e considerados significativos valores de $\mathrm{p}<0,05$ e intervalos de confiança de 95\% (IC95\%) maiores ou menores que 1 .

\section{RESULTADOS}

Na Tabela 1 se observa a distribuição dos participantes por características sociodemográficas, divididos em grupos de continentes e incontinentes. Participaram 238 longevos, $73 \%$ mulheres, $67 \%$ viúvos, $56 \%$ incontinentes, com média etária maior entre os incontinentes, $92,4 \pm 3,69$ anos $(p=0,299)$. Tanto o sexo quanto o estado conjugal apresentaram relação significativa com a perda urinária, sendo que as mulheres e os viúvos apresentaram maior frequência de perda urinária $(p<0,05)$. Com relação aos indicadores clínicos entre continentes e incontinentes, identificou-se que a média do escore de sintomas depressivos foi maior entre os incontinentes $(1,6 \pm 1,17$ sintomas, $p=0,052)$.

Na Tabela 2 se observa a distribuição dos continentes e incontinentes pelo grau de facilidade em executar as atividades indicativas de força e resistência muscular de membros inferiores e o tempo de duração do TUG. A dificuldade em levantar-se da cadeira, subir escadas e caminhar 400 metros foram relacionadas significativamente com a IU $(p<0,05)$. Participantes incontinentes apresentaram maior frequência de dificuldade e impossibilidade de executar as tarefas quando somadas as duas categorias. $\mathrm{O}$ tempo médio de realização do TUG não foi significativamente diferente entre os dois grupos. Entretanto, os continentes apresentaram um menor tempo de realização do TUG $(20,6 \pm 20,69$ s $)$ quando comparados aos incontinentes $(21,0 \pm 17,38 \mathrm{~s})$, representando uma maior velocidade de realização do teste.

Tabela 1. Distribuição dos participantes por características sociodemográficas e clínicas nos grupos continentes e incontinentes, $\mathrm{n}=238$, Porto Alegre, RS, Brasil, 2016.

\begin{tabular}{|c|c|c|c|c|}
\hline & $\begin{array}{c}\text { Continentes } \\
\text { n }(\%)\end{array}$ & $\begin{array}{c}\text { Incontinentes } \\
\text { n }(\%)\end{array}$ & $\begin{array}{l}\text { Total } \\
\text { n (\%) }\end{array}$ & $p$ \\
\hline \multicolumn{5}{|l|}{ Sexo } \\
\hline Feminino & $64(35)$ & $110(65)$ & $174(73)$ & \multirow{2}{*}{$0,001 *$} \\
\hline Masculino & $40(62)$ & $24(38)$ & $64(27)$ & \\
\hline \multicolumn{5}{|l|}{ Idade (anos) } \\
\hline Média $\pm \mathrm{DP}$ & $91,9 \pm 3,32$ & $92,4 \pm 3,69$ & $92,2 \pm 3,53$ & $0,299 \dagger$ \\
\hline \multicolumn{5}{|l|}{ Estado conjugal } \\
\hline Casado & $32(63)$ & $19(27)$ & $51(21)$ & \multirow{3}{*}{$0,017 *$} \\
\hline Solteiro/Divorciado & $13(48)$ & $14(52)$ & $27(11)$ & \\
\hline Viúvo & $59(37)$ & $101(63)$ & $160(67)$ & \\
\hline Escore dos sintomas depressivos & $1,4 \pm 1,09$ & $1,6 \pm 1,17$ & $229(96)$ & $0,052 \dagger$ \\
\hline TOTAL & $104(44)$ & $134(64)$ & $238(100)$ & \\
\hline
\end{tabular}

Nota: Ponto de corte de 2 pontos para sintomas depressivos.

* Teste de qui-quadrado; $\dagger$ teste de $t$ de Student. 
Tabela 2. Distribuição dos continentes e incontinentes pelo grau de facilidade e velocidade em executar as atividades indicadoras de força e resistência muscular de membros inferiores, $\mathrm{n}=238$, Porto Alegre, RS, Brasil, 2016.

\begin{tabular}{|c|c|c|c|c|}
\hline & $\begin{array}{c}\text { Continentes } \\
\text { n (\%) }\end{array}$ & $\begin{array}{c}\text { Incontinentes } \\
\mathbf{n}(\%)\end{array}$ & $\begin{array}{l}\text { Total } \\
\text { n (\%) }\end{array}$ & $p^{*}$ \\
\hline \multicolumn{5}{|c|}{ Grau de facilidade em levantar da cadeira $\dagger$} \\
\hline Não consegue & $21(20)$ & $56(42)$ & $77(32)$ & \multirow{4}{*}{0,001} \\
\hline Difícil & $31(30)$ & $30(22)$ & $61(26)$ & \\
\hline Mais ou menos fácil & $12(12)$ & $19(14)$ & $31(13)$ & \\
\hline Fácil & $39(38)$ & $29(22)$ & $68(29)$ & \\
\hline \multicolumn{5}{|c|}{ Grau de facilidade em caminhar 400 metros } \\
\hline Não consegue & $12(12)$ & $40(30)$ & $52(22)$ & \multirow{4}{*}{0,040} \\
\hline Difícil & $37(36)$ & $46(34)$ & $83(35)$ & \\
\hline Mais ou menos fácil & $22(21)$ & $22(16)$ & $44(18)$ & \\
\hline Fácil & $33(32)$ & $26(19)$ & $59(25)$ & \\
\hline \multicolumn{5}{|c|}{ Grau de facilidade em subir escadas $\dagger$} \\
\hline Não consegue & $13(12)$ & $40(30)$ & $53(22)$ & \multirow{4}{*}{0,011} \\
\hline Difícil & $37(36)$ & $36(27)$ & $73(31)$ & \\
\hline Mais ou menos fácil & $20(19)$ & 25 (19) & $45(19)$ & \\
\hline Fácil & $34(33)$ & $32(24)$ & $66(28)$ & \\
\hline \multicolumn{5}{|l|}{ TUG (segundos) } \\
\hline média $\pm \mathrm{DP}$ & $20,6 \pm 20,69$ & $21,0 \pm 17,38$ & & 0,874 \\
\hline
\end{tabular}

TUG = Timed Up and Go test.

* Teste de ANOVA; † Uma pessoa não soube responder.

A regressão logística foi utilizada para calcular as chances de o longevo apresentar IU. Em um modelo completo (Tabela 3 ) apenas a variável sexo continuava apresentando significância estatística. Por isso, foram criados modelos ajustados pelo sexo, para identificar a influência dessa variável sobre cada um dos fatores de risco identificados. Nesse novo modelo, apenas a facilidade de levantar da cadeira, caminhar 400 metros e o tempo do TUG continuavam significativos utilizando como referência ter algum grau de dificuldade, indicando efeito significativo sobre a continência independente do sexo. Nas regressões univariadas o sexo foi a variável com nível de significância maior. Também foram variáveis significativas, o estado conjugal, a dificuldade em levantar da cadeira, caminhar 400 metros e subir escadas.

Tabela 3. Regressões logísticas univariada e ajustada pelo sexo para a chance dos longevos apresentarem incontinência urinária, n=238, Porto Alegre, RS, Brasil, 2016.

\begin{tabular}{|c|c|c|c|c|c|c|}
\hline & \multicolumn{3}{|c|}{ Regressões univariadas } & \multicolumn{3}{|c|}{ Regressão ajustada pelo sexo* } \\
\hline & RC & IC $95 \%$ & $p$ & RC & IC $95 \%$ & $p$ \\
\hline Sexo masculino & 0,35 & $0,19-0,63$ & 0,001 & - & - & - \\
\hline Idade (anos) & 1,07 & $0,91-1,25$ & 0,377 & 0,34 & $0,78-1,12$ & 0,223 \\
\hline \multicolumn{7}{|l|}{ Estado conjugal (ref. Casado) } \\
\hline Solteiro/divorciado & 1,81 & $0,71-4,66$ & 0,217 & 1,12 & $0,39-3,16$ & 0,836 \\
\hline Viúvo(a) & 2,88 & $1,50-5,54$ & 0,002 & 1,85 & $0,87-3,94$ & 0,893 \\
\hline Sintomas depressivos & 0,56 & $0,33-0,96$ & 0,034 & 0,60 & $0,35-1,04$ & 0,069 \\
\hline $\begin{array}{l}\text { Facilidade em levantar-se da cadeira } \\
\text { (ref. dificuldade) }\end{array}$ & 0,83 & $0,73-0,94$ & 0,003 & 0,85 & $0,74-0,96$ & 0,012 \\
\hline $\begin{array}{l}\text { Facilidade em caminhar } 400 \text { metros } \\
\text { ref. dificuldade }\end{array}$ & 0,81 & $0,70-0,92$ & 0,002 & 0,83 & $0,72-0,96$ & 0,001 \\
\hline $\begin{array}{l}\text { Facilidade em subir escadas } \\
\text { ref. dificuldade }\end{array}$ & 0,87 & $0,76-0,99$ & 0,004 & 0,90 & $0,79-1,04$ & 0,105 \\
\hline TUG & 1,00 & $0,99-1,02$ & 0,890 & 0,352 & $0,18-0,69$ & 0,002 \\
\hline
\end{tabular}

TUG = Timed Up and Go Test; RC = Razão de chance; IC = Intervalo de confiança; $p=$ nível de significância. Significativo estatisticamente quando $p<0,05$.

* Regressões univariadas ajustadas pelo sexo para cada variável. 
Tabela 4. Regressões logísticas univariada para homens e mulheres para a chance dos longevos apresentarem incontinência urinária, n=238, Porto Alegre, RS, Brasil, 2016.

\begin{tabular}{|c|c|c|c|c|c|c|}
\hline & \multicolumn{3}{|c|}{ Regressões univariadas para homens } & \multicolumn{3}{|c|}{ Regressão univariada para mulheres } \\
\hline & RC & IC $95 \%$ & $p$ & RC & IC 95\% & $p$ \\
\hline Idade (anos) & 1,07 & $0,92-1,26$ & 0,377 & 1,01 & $0,93-1,11$ & 0,743 \\
\hline \multicolumn{7}{|l|}{ Estado conjugal (ref. Casado) } \\
\hline Outro & 1,18 & $0,10-14,42$ & 0,895 & 0,89 & $0,23-3,35$ & 0,858 \\
\hline Viúvo(a) & 2,36 & $0,81-6,86$ & 0,113 & 1,42 & $0,46-4,33$ & 0,537 \\
\hline $\begin{array}{l}\text { Sintomas depressivos } \\
\text { (ref. }<2 \text { pontos) }\end{array}$ & 0,33 & $0,11-0,97$ & 0,044 & 0,74 & $0,40-1,40$ & 0,359 \\
\hline Facilidade em levantar-se da cadeira & 0,90 & $0,70-1,17$ & 0,464 & 0,82 & $0,71-0,96$ & 0,012 \\
\hline Facilidade em caminhar 400 metros & 0,84 & $0,63-1,10$ & 0,207 & 0,83 & $0,70-0,98$ & 0,027 \\
\hline Facilidade em subir escadas & 1,12 & $0,85-1,47$ & 0,395 & 0,84 & $0,71-0,99$ & 0,029 \\
\hline TUG & 1,05 & $0,99-1,13$ & 0,105 & 0,10 & $0,98-1,01$ & 0,454 \\
\hline
\end{tabular}

TUG $=$ Timed Up and Go Test; RC = Razão de chance; IC = Intervalo de confiança; $p=$ nível de significância. Significativo estatisticamente quando $p<0,05$.

A Tabela 4 apresenta as razões de chance das regressões logísticas univariadas calculadas para homens e mulheres para a chance de apresentar incontinência urinária na presença de cada uma das situações investigadas (variáveis independentes). Observou-se que na regressão univariada para homens a única variável significativa que poderia influenciar a chance de apresentar IU foram os sintomas depressivos $(p=0,044)$. Já nas mulheres, a facilidade em levantarse da cadeira, caminhar 400 metros e subir escadas foram variáveis com associação significativa à chance de desenvolver IU Dessa forma, podemos inferir que as alterações funcionais, relacionada a força e resistência dos MMII, estão mais relacionadas à IU em mulheres do que nos homens.

\section{DISCUSSÃO}

O presente trabalho teve o intuito de investigar a existência de possíveis diferenças entre os sexos, a relação com os indicadores de força/resistência muscular de MMII e a presença de IU em nonagenários e centenários, valendo-se que os achados poderiam ajudar o tratamento dessa disfunção. A literatura científica demonstra a importância de verificar outros grupos musculares, como os abdominais, e sua relação com a musculatura do assoalho pélvico. ${ }^{17}$ Devido à sinergia muscular e seu funcionamento em cadeias, podemos relacionar o funcionamento da musculatura do assoalho pélvico e músculos abdominais com os achados do presente estudo, que demonstram a relação entre a IU e indicadores de força e resistência muscular em MMII. Considerando que a IU é uma síndrome geriátrica, a diminuição da força muscular pode ser considerada um sinal de alerta, tanto para prevenção, quanto para o seu tratamento. ${ }^{18}$

A frequência de IU é maior em mulheres e aumenta com a idade ${ }^{18}$. Em nosso estudo as mulheres apresentaram maior frequência de perda urinária (65\%) quando comparado aos homens (38\%). A literatura explica essa maior frequência em mulheres em função de uma desvantagem anatômica, em que a bexiga é menor, canal da uretra é mais curto do que dos homens e também os órgãos pélvicos se localizam acima da bexiga, aumentando a compressão. ${ }^{19}$ Além disso, a mulher é mais exposta a fatores de risco como obesidade, paridade (tipo de parto, uso anestesia no parto, peso do recém-nascido), menopausa, cirurgias ginecológicas, constipação intestinal, maior número de doenças crônicas, menores níveis de prática de atividade física e fatores hereditários, que estão intimamente relacionados com o enfraquecimento da musculatura pélvica. ${ }^{18}$ As principais causas de IU no sexo masculino são a Hiperplasia Benigna Prostática e as neoplasias prostáticas ${ }^{20,21}$ que não foram investigadas no estudo, sendo uma limitação deste. Sendo assim, embora não tenhamos avaliado a presença dessas afecções, sugere-se que esta porcentagem inferior de homens incontinentes se deve possivelmente à não existência dessas patologias.

Observou-se nesse estudo que a frequência de IU foi maior que o encontrado na literatura em ambos os sexos. Esse achado pode ser explicado pois a idade dos participantes dessa pesquisa em questão é maior que a das demais encontradas. São vários os fatores de risco encontrados na literatura que se associam e contribuem para o desenvolvimento da IU, entre eles, o envelhecimento natural das fibras musculares do assoalho pélvico. ${ }^{12}$ 
Os incontinentes foram o grupo que contou com mais participantes viúvos. Este achado corrobora com uma pesquisa que ao avaliar a IU e a funcionalidade de uma população idosa, encontrou relação significativa entre a IU e estado civil $(p=0,037)$, apontando que entre os incontinentes, $73,8 \%$ eram viúvos. ${ }^{22} \mathrm{Um}$ resumo publicado na revista The Journal of Urology em 2016 encontrou efeito protetor para incontinência urinária ser casado e os autores relataram ser um novo achado para a literatura. ${ }^{23} \mathrm{~A}$ justificativa para essa relação carece de estudos pois não foi encontrada na literatura.

Com relação à presença de sintomas depressivos medida pela Escala de Depressão Geriátrica de 5 questões, os incontinentes apresentaram uma pontuação maior quando comparado com os continentes. Tal achado corrobora com um trabalho que avaliou a qualidade de vida de mulheres com IU e observou que quase $41,9 \%$ da amostra que tinha limitação funcional em virtude da IU manifestou problemas emocionais como depressão. ${ }^{24}$ Uma outra pesquisa avaliando a qualidade de vida, relata que normalmente, a IU gera perda de autoconfiança e autoestima, levando ao isolamento social e sentimentos como solidão e tristeza que consequentemente geram sintomas depressivos. ${ }^{25,26}$

A variável viuvez em outro estudo foi influenciada pela presença da depressão. Relacionando com as demais variáveis estudadas como o sexo e o estado civil, mulheres casadas incontinentes podem ter pior qualidade de vida, quando comparadas com as não casadas com os mesmos sintomas..$^{25}$ Esse fato pode ser explicado pela presença de depressão ${ }^{26}$, que de acordo com achados de outra pesquisa, poderia ser uma consequência da $\mathrm{IU}^{27}$. Além disso, a IU pode influenciar negativamente a autopercepção de saúde ${ }^{12,28}$ e consequentemente mascararem o relato do grau de dificuldade para realizar atividades de mobilidade e força de MMII. ${ }^{10,26}$ No presente estudo os sintomas depressivos foram particularmente mais importantes entre os homens avaliados. Outro estudo corrobora com a discussão ao evidenciar que a IU afetaria negativamente a vida dos homens, principalmente com relação a suas parceiras, especialmente nas questões emocionais, sexuais e sociais, destacando-se o sofrimento psíquico que eles geram. ${ }^{10} \mathrm{~A}$ IU foi mensurada por autorrelato, e dessa forma, muitos homens poderiam ter omitido a presença desse problema durante a avaliação, por vergonha ou constrangimento, principalmente na presença de suas companheiras.

Foi constatado que a maioria dos incontinentes apresentou algum nível de dificuldade para levantar de uma cadeira, caminhar e subir escadas e esse percentual foi menor nos continentes nas três atividades sugerin- do a eles uma melhor funcionalidade, sendo mais determinantes entre as mulheres. Em outra pesquisa, na associação entre IU e funcionalidade também se observou escores mais baixos de funcionalidade no grupo de incontinentes. Os escores funcionais baixos identificados nas atividades básicas e instrumentais de vida diária, como também da locomoção foram descritos como precipitantes da IU.22

Foi identificado nas análises que embora ambos os grupos se enquadraram na classificação de alto risco de quedas, a partir do TUG, os incontinentes apresentaram uma média de segundos superior na realização do teste, sugerindo uma relação entre IU e quedas, porém sem diferença estatística. O TUG é um teste de mobilidade frequentemente utilizado para se avaliar o risco de queda. ${ }^{28,29}$

Continentes bem como incontinentes apresentaram alto tempo de execução do TUG, condizente com alto risco de quedas, sendo a média maior nos incontinentes, embora não significativa. Alguns estudos sugerem existir uma necessidade do idoso urinar com maior frequência e a incapacidade de adiamento da micção e preocupação de não urinar na roupa que o obrigaria a ir ao banheiro mais vezes que o esperado, expondo-o ao maior risco de quedas. Da mesma forma, as deficiências nos sistemas visual, vestibular e somatossensorial que ocorrem com o envelhecimento, poderiam levar a dificuldade de equilíbrio e marcha. Essas disfunções, concomitante à IU poderiam resultar em queda..$^{30,31}$

Os resultados alcançados são importantes pois favorecem o desenvolvimento de ações preventivas e intervenções para a IU, desfavorecendo a sua progressão. Especialmente nos aspectos da saúde do idoso, como a funcionalidade e qualidade de vida. ${ }^{32,33}$ Porém, algumas limitações desse estudo devem ser destacadas, como a variável dependente (IU) ter sido coletada por meio de autorrelato e a não abordagem dos diferentes tipos de IU, que poderiam ter relações diferentes com a musculatura. Contudo, espera-se que as limitações apontadas sejam objetivos para estudos futuros com a mesma população estudada.

\section{CONCLUSÃO}

Os indicadores de força e resistência muscular em MMII apresentaram relação positiva com a chance de apresentar IU e homens e mulheres apresentaram determinantes de IU diferentes. Esses resultados reforçam a necessidade da abordagem da IU também no sexo masculino, mesmo que as mulheres a desenvolvam com maior frequência. A IU foi um fator significativamente relacionado a sintomas depressivos nos homens, fato esse pouco descrito na literatura 
atual. Investigando mais profundamente a diferença entre homens e mulheres, foi possível identificar que na relação entre IU e força/resistência muscular de MMII, a ausência de sintomas depressivos era um fator de proteção, enquanto que, nas mulheres os determinantes de força/resistência muscular de MMII utilizados no estudo, eram os fatores de proteção. $\mathrm{Na}$ prática, os resultados demonstram que em atividades de prevenção, exercícios de força e ressitência muscular de membros MMII podem ser agregados aos exercícios de força da musculatura do assoalho pélvico para atenuar que os nonagenários e centenários e, principalmente as mulheres desenvolvam a IU. Também se faz importante enfatizar a abordagem multiprofissional dos pacientes com IU, particularmente os homens nonagenários e centenários que apresentam importantes sintomas depressivos.

\section{AGRADECIMENTOS}

O presente trabalho foi realizado com apoio da Coordenação de Aperfeiçoamento de Pessoal Nível Superior - Brasil (CAPES) - Código de Financiamento 001.

\section{REFÊRENCIAS}

1. International Continence Society. Recommendations of the International Scientific Committee: evaluation and treatment of urinary incontinence, Pelvic Organ Prolapse and Faecal Incontinence. 4aㅡ International Consultation on Incontinence; 2008; Paris. Paris, França: ICUD; 2009 [acesso em 30 set 2018]. [aproximadamente 54p.]. Disponível em: http:// www.ics.org/Publications/ICI 4/filesbook/recommendation.pdf

2. Milsom I, Coyne, KS, Nicholson S, et al. Global Prevalence and Economic Burden of Urgency Urinary Incontinence: A Systematic Review. European Urology. 2014; 65(1):79-95.

3. Santos PHS, Fernandes MH, Casotti CA, et al. Perfil de fragilidade e fatores associados em idosos cadastrados em uma Unidade de Saúde da Família. Ciênc. Saúde Coletiva. 2015;20(6):1917-24.

4. Lenardt MH, Grden CRB, Sousa JAV, et al. Fatores associados à diminuição de força de preensão manual em idosos longevos Rev. Esc. Enferm. USP 2014;48(6):1006-12.

5. Ângela Kemel Zanella. Avaliação da consciência da musculatura do assoalho pélvico e sua relação com a incontinência urinária em idosas [tese]. Porto Alegre (RS): Pontifícia Universidade Católica do Rio Grande do Sul; 2016.

6. Berardelli M, De Rango F, Morelli M, et al. Urinary incontinence in the elderly and in the oldest old: correlation with frailty and mortality. Rejuvenation Res. 2013;16(3): 206-11.

7. Jenkins KR, Fultz NH. Functional impairment as a risk factor for urinary incontinence among older Americans. Neurourol Urodyn. 2005;24(1):51-5.

8. Hayashida I, Tanimoto Y, Takahashi Y, et al. Correlation between muscle strength and muscle mass, and their association with walking speed, in community-dwelling elderly Japanese individuals. Plos One. 2014;9(11): e111810.
9. Gabriela Guimarães Oliveira. Desempenho de longevos caidores e não caidores na avaliação do Timed up and go (TUG) utilizando um aplicativo de smartphone [dissertação]. Porto Alegre: Pontifícia Universidade Católica do Rio Grande do Sul; 2018.

10. Bicalho MB, Lopes MHBM. Impacto da incontinência urinária na vida de esposas de homens com incontinência: revisão integrativa. Rev. Esc. Enferm. USP. 2012;46(4): 1009-14.

11. Pícoli, T da S, Figueiredo, LL de, Patrizzi, LJ. Sarcopenia e envelhecimento. Fisioter. Mov. 2011; 24(3):455-62.

12.Sousa JG, Ferreira VR, Oliveira RJ et al. Avaliação da força muscular do assoalho pélvico em idosas com incontinência urinária. Fisioter. Mov. 2011;24(1):39-46.

13. Observatório da Cidade de Porto Alegre, OBSERVAPOA. Porto Alegre em mapas: territorialidades: regiões OP [Internet]. Porto Alegre; 1997. [Acesso em 30 Set 2018]. Disponível em: http://www.observapoa.com.br/default. php?p_secao $=46$

14. Podsiadlo D, Richardson S. The timed "Up \& Go": a test of basic functional mobility for frail elderly persons. J Am Geriatr Soc. 1991;39(2):142-8.

15. Marília Siqueira Campos Almeida. Efetividade da escala de depressão geriátrica de cinco itens em população idosa da comunidade [tese]. Porto Alegre: Pontifícia Universidade Católica do Rio Grande do Sul; 2011.

16. Apóstolo JLA, Loureiro LMJ, Reis IAC, et al. Contribuição para a adaptação da Geriatric Depression Scale-15 para a língua portuguesa. Rev. Enf. Ref. 2014;ser IV(3): 65-73.

17. Korelo RIG, Kosiba CR, Grecco L, et al. Influência do fortalecimento abdominal na função perineal, associado ou não à orientação de contração do assoalho pélvico, em nulíparas. Fisioter. Mov. 2011;24(1):75-85.

18. Higa R, Lopes MHBM, Reis MJ. Fatores de risco para incontinência urinária na mulher. Rev. Esc. Enferm. USP. 2008;42(1):187-92.

19. Neto OMV. Infecção do trato urinário. RMRP. 2003; 36(2/4):365-9.

20. Bing MT, Uhlman MA, Kreder KJ. An update in the treatment of male urinary incontinence. Curr. Opin. Urol. 2013;23(6):540-4.

21. Kubagawa LM, Pellegrini JRF, Lima VP, et al. A eficácia do tratamento fisioterapêutico da incontinência urinária masculina após prostatectomia. Rev. Bras. Cancerol. 2006; 52(2):179-83.

22. Fontes AP, Botelho MA, Fernandes AA. Incontinência Urinária e Funcionalidade: um estudo exploratório numa população idosa. Acta Urológica. 2011;2:12-9.

23. Kreydin E, Kim M, Oliver J, et al. MP77-11 til death do us part: the relationship between urinary incontinence and marital status among us women and men. The Journal of Urology. 2016;195(4): e1020.

24. Pedro AF, Ribeiro J, Soler ZASG, et al. Qualidade de vida de mulheres com incontinência urinária. SMAD, Rev. Eletrônica Saúde Mental Álcool Drog. (Ed. port.). 2011;7(2):63-70.

25. Knorst MR, Resende TL, Goldim JR. Perfil clínico, qualidade de vida e sintomas depressivos de mulheres com incontinência urinária atendidas em hospital-escola. Rev. Bras. Fisioter. 2011;15(2):109-16.

26. Hellwig N, Munhoz TN, Tomasi E. Sintomas depressivos em idosos: estudo transversal de base populacional. Ciênc. Saúde Coletiva. 2016;21(11):3575-84. 
27. Vigod SN, Stewart DE. Major depression in female urinary incontinence. Psychosomatics. 2006;47(2):147-51.

28. Bischoff HA, Stähelin HB, Monsch AU, et al. Identifying a cut-off point for normal mobility: a comparison of the timed 'up and go' test in community-dwelling and institutionalised elderly women. Age Ageing. 2003;32(3):315-20.

29. Bolina AF, Dias FA, Santos NMF, et al. Incontinência urinária autorreferida em idosos e seus fatores associados. Rev. Rene. 2003;14(2):354-63.

30. Abreu HCA, Reiners AAO, Azevedo RCS, et al. Incontinência urinária na predição de quedas em idosos hospitalizados. Ver. Esc. Enferm. USP. 2014;48(5):851-6.
31. Rios AA, Cardoso JR, Rodrigues MA, et al. The helpseeking by women with urinary incontinence in Brazil. Int Urogynecol. J. 2011;22(7):879-84.

32. Castaneda L, Plácido T. Ligação do King’s Heath Questionário com a Classificação Internacional de Funcionalidade, Incapacidade e Saúde, para avaliação de pacientes com incontinência urinária pós cirurgia oncológica ginecológica. Acta Fisiatr. 2010;17(1):18-21.

33. Depolito C, Leocadio PLLF, Cordeiro RC. Declínio funcional de idosa institucionalizada: aplicabilidade do modelo da Classificação Internacional de Funcionalidade, Incapacidade e Saúde. Fisioter. Pesqui. 2009;16(2):23-9.

\section{AUTORES:}

LUISA BRAGA JORGE <lbragajorge@hotmail.com>

Fisioterapeuta. Mestre em Gerontologia Biomédica. Doutoranda do Programa de Pós-graduação em Gerontologia Biomédica da Escola de Medicina da Pontifícia Universidade Católica do Rio Grande do Sul.

(D) orcid.org/0000-0003-2695-8340

JOSEMARA DE PAULA ROCHA <josemara.rocha@hotmail.com>

Fisioterapeuta. Mestre em Envelhecimento Humano. Especialização em Residência Multiprofissional em Saúde do Idoso. Doutoranda do Programa de Pós-graduação em Gerontologia Biomédica da Escola de Medicina da Pontifícia Universidade Católica do Rio Grande do Sul.

PATRICIA MORSCH <patriciamorsch@hotmail.com>

Fisioterapeuta. Especialização Acadêmica em Saúde Pública. Mestre em gerontologia (University of North Carolina/Charlotte/EUA).

Doutora em Gerontologia Biomédica. Docente e coordenadora do Curso de Fisioterapia da Faculdade de Educação e Meio Ambiente (FAEMA).

BRUNA BORBA NEVES <brunanevesto@hotmail.com>

Terapeuta ocupacional. Mestre em Gerontologia Biomédica. Doutoranda do Programa de Pós-graduação em Gerontologia Biomédica da Escola de Medicina da Pontifícia Universidade Católica do Rio Grande do Sul.

GABRIELA GUIMARÃES OLIVEIRA <oliveira_gabriela@hotmail.com>

Fisioterapeuta. Mestre em Gerontologia Biomédica. Especialização em Residência Multiprofissional em Saúde do Idoso. Doutoranda do Programa de Pós-graduação em Gerontologia Biomédica da Escola de Medicina da Pontifícia Universidade Católica do Rio Grande do Sul.

ÂNGELO JOSÉ GONÇALVES BÓS <angelo.bos@pucrs.br>

Médico. Residências em Saúde Comunitária e em Geriatria e Gerontologia. Especialista em Geriatria pela Sociedade Brasileira de Geriatria e Gerontologia. Doutorado em Saúde Comunitária. Pós-doutorado em Epidemiologia do Envelhecimento (Escola de Saúde Pública/Johns Hopkins/Baltimore/Estados Unidos) , em Estudos Longitudinais (Instituto Nacional em Envelhecimento/Baltimore/Estados Unidos) e em Saúde e Participação Social (Instituto Metropolitano de Geriatria e Gerontologia/Tóquio/Japão). Docente da Faculdade de Medicina e do Programa de Pós-graduação em Gerontologia Biomédica da Escola de Medicina da Pontifícia Universidade Católica do Rio Grande do Sul. 